\title{
TAME AND PURELY WILD EXTENSIONS OF VALUED FIELDS
}

\author{
YU. L. ERSHOV
}

Dedicated to the centenary of the birth of the outstanding Russian mathematician D. K. Faddeev

\begin{abstract}
A systematic and concise exposition of the basic results concerning two complementary classes (tame and purely wild) of extensions of (Henselian) valued fields is given. These notions proved to be quite useful both for the general theory and for the model theory of such fields. Along with new results, new proofs of old results are presented. Thus, in the proof of the well-known Pank theorem on the existence of a complement to the ramification group in the absolute Galois group of a Henselian valued field, the properties of maximal immediate extensions are employed instead of cohomological methods.
\end{abstract}

Let $F$ be a field. By definition, a valuation ring of $F$ is an arbitrary subring $R$ of $F$ such that, for each $a \in F^{\times}=F \backslash\{0\}$, we have either $a \in R$ or $a^{-1} \in R$. Every valuation $\operatorname{ring} R$ is a local ring; i.e., it has a unique maximal ideal $\mathfrak{m}(R)$. The quotient ring $F_{R} \rightleftharpoons R / \mathfrak{m}(R)$ of $R$ by the maximal ideal $\mathfrak{m}(R)$ is called the residue field. The quotient group $\Gamma_{R} \rightleftharpoons F^{\times} / U(R)$ of the multiplicative group $F^{\times}$of the field $F$ by the multiplicative group $U(R)$ of units (invertible elements) of $R$ is called the valuation group. The group $\Gamma_{R}$ is equipped with the structure of an ordered group in which the cone of nonnegative elements is the image of the set $R \backslash\{0\}$ in $\Gamma_{R}$. We use the additive notation for the operation in $\Gamma_{R}$.

The homomorphism $v_{R}: F^{\times} \rightarrow \Gamma_{R}=F^{\times} / U(R)$ is called the valuation (determined by the valuation $\operatorname{ring} R$ ).

A pair $\mathbb{F}=\langle F, R\rangle$, where $F$ is a field and $R$ is a valuation ring of $F$, is called a valued field. If $\mathbb{F}=\langle F, R\rangle, \mathbb{F}_{0}=\left\langle F_{0}, R_{0}\right\rangle$ are valued fields, $F \leq F_{0}$, and $R_{0} \cap F=R$, then $\mathbb{F}_{0}$ is called an extension of $\mathbb{F}\left(\mathbb{F} \leq \mathbb{F}_{0}\right)$. If $\mathbb{F} \leq \mathbb{F}_{0}$, then the residue field $F_{R}$ is naturally identified with a subfield of the residue field $F_{R_{0}}\left(F_{R} \leq F_{R_{0}}\right)$, and the valuation group $\Gamma_{R}$ is naturally identified with a subgroup of $\Gamma_{R_{0}}\left(\Gamma_{R} \leq \Gamma_{R_{0}}\right)$.

A valued field $\mathbb{F}$ is said to be Henselian if, for every algebraic extension $F_{0} \geq F$, there exists a unique valuation ring $R_{0}$ in $F_{0}$ such that $\left\langle F_{0}, R_{0}\right\rangle \geq \mathbb{F}$.

A valued field $\mathbb{F}=\langle F, R\rangle$ is Henselian if and only if the following statement is valid.

Hensel lemma. Let $f \in R[x]$ be a monic polynomial such that the image $\bar{f}$ of $f$ in $F_{R}[x]$ has a simple root $\bar{\alpha}$ in $F_{R}$. Then the polynomial $f$ has a root $\alpha \in R$ such that $\bar{\alpha}=\alpha+\mathbf{m}(R)$.

If $\mathbb{F}_{0} \geq \mathbb{F}$ is a finite extension (i.e., $\left[F_{0}: F\right]<\omega$ ) of valued fields, then the degree $f \rightleftharpoons\left[F_{R_{0}}: F_{R}\right]$ of the extension $F_{R_{0}} \geq F_{R}$ and the index $e \rightleftharpoons\left[\Gamma_{R_{0}}: \Gamma_{R}\right]$ of the subgroup $\Gamma_{R}$ in $\Gamma_{R_{0}}$ are finite and we have the following basic inequality: $e \cdot f \leq n \rightleftharpoons\left[F_{0}: F\right]$.

2000 Mathematics Subject Classification. Primary 12F15.

Key words and phrases. Henselian valued fields, valuation ring, valuation group, ramified extension, totally unramified extension.

Partially supported by the Council on Grants of President of the Russian Federation for the state support of leading scientific schools (project no. NSh-4787.2006.1). 
A valued field $\mathbb{F}$ is said to be algebraically complete if, for every finite extension $\mathbb{F}_{0} \geq \mathbb{F}$, equality occurs in the basic inequality: $n=e \cdot f$.

An extension $\mathbb{F}_{0} \geq \mathbb{F}$ of valued fields is immediate if $F_{R_{0}}=F_{R}$ and $\Gamma_{R_{0}}=\Gamma_{R}$.

A finite extension $\mathbb{F}_{0} \geq \mathbb{F}$ is unramified (respectively, totally ramified) if $\left[F_{0}: F\right]=$ $\left[F_{R_{0}}: F_{R}\right](=f)$ (respectively, $\left.\left[F_{0}: F\right]=\left[\Gamma_{R_{0}}: \Gamma_{R}\right]=e\right)$.

We say that an arbitrary algebraic extension $\mathbb{F}_{0} \geq \mathbb{F}$ is unramified (respectively, totally ramified) if, for every intermediate extension $\left(\mathbb{F}_{0} \geq\right) \mathbb{F}_{1} \geq \mathbb{F}$ finite over $\mathbb{F}$, the extension $\mathbb{F}_{1} \geq \mathbb{F}$ is unramified (respectively, totally ramified).

In what follows, we fix a Henselian valued field $\mathbb{F}=\langle F, R\rangle$ such that the residue field $F_{R}$ has characteristic $p>0$.

A finite extension $\mathbb{F}_{0} \geq \mathbb{F}$ is said to be tame if the following conditions are fulfilled:

1) $F_{R_{0}}$ is a separable extension of $F_{R}$;

2) $p$ does not divide the index $\left[\Gamma_{R_{0}}: \Gamma_{R}\right]$;

3) the extension $\mathbb{F}_{0} \geq \mathbb{F}$ is defectless, i.e., $\left[F_{0}: F_{1}\right]=\left[F_{R_{0}}: F_{R}\right] \cdot\left[\Gamma_{R_{0}}: \Gamma_{R}\right]$.

An algebraic extension $\mathbb{F}_{0} \geq \mathbb{F}$ is tame (see [3, 4]) if, for every intermediate subextension $\left(\mathbb{F}_{0} \geq\right) \mathbb{F}_{1} \geq \mathbb{F}$ finite over $\mathbb{F}\left(\left[F_{1}: F\right]<\omega\right)$, the extension $\mathbb{F}_{1} \geq \mathbb{F}$ is tame.

Corollary 1 (to the definition). Let $\mathbb{F}_{0} \geq \mathbb{F}_{1} \geq \mathbb{F}$ be a tower of algebraic extensions. The extension $\mathbb{F}_{0} \geq \mathbb{F}$ is tame if and only if the extensions $\mathbb{F}_{0} \geq \mathbb{F}_{1}$ and $\mathbb{F}_{1} \geq \mathbb{F}$ are tame.

For finite extensions, this follows directly from the relations $F_{R_{0}} \geq F_{R_{1}} \geq F_{R},\left[F_{R_{0}}\right.$ : $\left.F_{R}\right]=\left[F_{R_{0}}: F_{R_{1}}\right]\left[F_{R_{1}}: F_{R}\right], \Gamma_{R_{0}} \geq \Gamma_{R_{1}} \geq \Gamma_{R}$, and $\left[\Gamma_{R_{0}}: \Gamma_{R}\right]=\left[\Gamma_{R_{0}}: \Gamma_{R_{1}}\right]\left[\Gamma_{R_{1}}: \Gamma_{R}\right]$.

Corollary 2 (to the definition). If $\mathbb{F}$ is algebraically complete, the residue field $F_{R}$ is perfect, and the group $\Gamma_{R}$ is p-divisible $\left(p \Gamma_{R}=\Gamma_{R}\right)$, then every algebraic extension $\mathbb{F}_{0} \geq \mathbb{F}$ is tame.

It is convenient to define the complementary notion of a purely wild extension not only for algebraic extensions. An extension $\mathbb{F}_{0} \geq \mathbb{F}$ is said to be purely wild (see [3, 4]) if $F_{R_{0}}$ is a purely inseparable extension of $F_{R}$ and the quotient group $\Gamma_{R_{0}} / \Gamma_{R}$ is a $p$-group.

Remark 1. Every immediate extension is purely wild.

Remark 2. If $\mathbb{F}_{0} \geq \mathbb{F}$ is a purely inseparable extension, then $\mathbb{F}_{0} \geq \mathbb{F}$ is a purely wild extension.

Corollary 3 (to the definition). Let $\mathbb{F}_{0} \geq \mathbb{F}_{1} \geq \mathbb{F}$ be a tower of extensions. The extension $\mathbb{F}_{0} \geq \mathbb{F}$ is purely wild if and only if the extensions $\mathbb{F}_{0} \geq \mathbb{F}_{1}$ and $\mathbb{F}_{1} \geq \mathbb{F}$ are purely wild.

This fact can be proved in the same way as Corollary 1.

Corollary 4 (to the definition). An extension $\mathbb{F}_{0} \geq \mathbb{F}$ is tame and purely wild if and only if $\mathbb{F}_{0}=\mathbb{F}$.

Indeed, such an extension $\mathbb{F}_{0} \geq \mathbb{F}$ must be immediate. Since it is defectless, we see that $\mathbb{F}_{0}=\mathbb{F}$.

Corollary 5. If $\mathbb{F}_{0} \geq \mathbb{F}$ is tame, then $F_{0}$ is a separable extension of $F$.

Proof. Indeed, let $F_{0, s}$ be the separable closure of $F$ in $F_{0}$, and let $\mathbb{F}_{0, s} \rightleftharpoons\left\langle F_{0, s}, R_{0} \cap F_{0, s}\right\rangle$. Then the extension $\mathbb{F}_{0} \geq \mathbb{F}_{0, s}$ is tame and purely wild simultaneously (Corollary 1 and Remark 2). By Corollary 3, we have $\mathbb{F}_{0}=\mathbb{F}_{0, s}$.

Proposition 1. Let $\mathbb{F}^{\prime} \geq \mathbb{F}_{i} \geq \mathbb{F}$ be extensions such that $\mathbb{F}_{0} \geq \mathbb{F}$ is tame and $\mathbb{F}_{0} \geq \mathbb{F}$ is purely wild. Then $F_{0}$ and $\bar{F}_{1}$ are linearly disjoint over $F\left(F_{0} \|_{F} \mathbb{F}_{1}\right)$, and for $F^{\prime \prime} \rightleftharpoons$ $F_{0} F_{1}=F_{0} \otimes_{F} F_{1}$ and $\mathbb{F}^{\prime \prime} \rightleftharpoons\left\langle F^{\prime \prime}, R^{\prime} \cap F^{\prime \prime}\right\rangle$ the extension $\mathbb{F}^{\prime \prime} \geq \mathbb{F}_{0}$ is purely wild and $\mathbb{F}^{\prime \prime} \geq \mathbb{F}_{1}$ is tame. 
Proof. Without loss of generality, we may assume that the extension $\mathbb{F}_{0} \geq \mathbb{F}$ is finite and $F^{\prime \prime}=F^{\prime}$. We have $F_{R} \leq F_{R_{i}} \leq F_{R^{\prime}}, i=0,1, F_{R_{0}}$ is a separable extension of $F_{R}$, and $F_{R_{1}}$ is a purely inseparable extension of $F_{R}$. Consequently, $F_{R} \leq F_{R_{0}} F_{R_{1}}=$ $F_{R_{0}} \otimes_{F_{R}} F_{R_{1}} \leq F_{R^{\prime}}$ and $\left[F_{R^{\prime}}: F_{R_{1}}\right] \geq\left[F_{R_{0}}: F_{R}\right]$. We have $\Gamma_{R} \leq \Gamma_{R_{i}} \leq \Gamma_{R^{\prime}}(i=0,1), p$ does not divide the index $\left[\Gamma_{R_{0}}: \Gamma_{R}\right]$, and $\Gamma_{R_{1}} / \Gamma_{R}$ is a p-group, whence $\Gamma_{R_{0}} \cap \Gamma_{R_{1}}=\Gamma_{R}$ and $\left[\Gamma_{R^{\prime}}: \Gamma_{R_{1}}\right] \geq\left[\Gamma_{R_{0}}: \Gamma_{R}\right]\left(\Gamma_{R^{\prime}} / \Gamma_{R_{1}} \geq \Gamma_{R_{0}} \Gamma_{R_{1}} / \Gamma_{R_{1}} \simeq \Gamma_{R_{0}} / \Gamma_{R_{0}} \cap \Gamma_{R_{1}}=\Gamma_{R_{0}} / \Gamma_{R}\right)$. Next, $\left[F_{R_{0}}: F_{R}\right] \cdot\left[\Gamma_{R_{0}}: \Gamma_{R}\right]=\left[F_{0}: F\right] \geq\left[F^{\prime}: F_{1}\right] \geq\left[F_{R^{\prime}}: F_{R_{1}}\right] \cdot\left[\Gamma_{R^{\prime}}: \Gamma_{R_{1}}\right] \geq\left[F_{R_{0}}:\right.$ $\left.F_{R}\right] \cdot\left[\Gamma_{R_{0}}: \Gamma_{R}\right]$. Consequently, $\left[F_{0}: F\right]=\left[F^{\prime}: F_{1}\right], F_{R^{\prime}}=F_{R_{0}} \otimes F_{R_{1}}, \Gamma_{R}^{\prime}=\Gamma_{R_{0}} \Gamma_{R_{1}}, F_{R^{\prime}}$ is a separable extension of $F_{R_{1}}$ and a purely inseparable extension of $F_{R_{0}},\left[\Gamma_{R^{\prime}}: \Gamma_{R_{1}}\right]=$ $\left[\Gamma_{R_{0}}: \Gamma_{R}\right]$, and $\Gamma_{R^{\prime}} / \Gamma_{R_{0}}=\Gamma_{R_{0}} \Gamma_{R_{1}} / \Gamma_{R_{0}}=\Gamma_{R_{1}} / \Gamma_{R_{1}} \cap \Gamma_{R_{0}}=\Gamma_{R_{1}} / \Gamma_{R}$ is a $p$-group. Now, all conclusions of the proposition follow from the relations obtained.

Proposition 2. Let $\mathbb{F}_{0} \geq \mathbb{F}$ be a finite extension, and let $\left(\mathbb{F}_{0} \geq\right) \mathbb{F}_{1} \geq \mathbb{F}$ be the maximal (with respect to inclusion) tame subextension of $\mathbb{F}_{0}$. Then $\mathbb{F}_{0} \geq \mathbb{F}_{1}$ is a purely wild extension.

Proof. We verify that $F_{R_{1}}$ coincides with the separable closure $F_{s}$ of $F_{R}$ in $F_{R_{0}}$. Indeed, since $\mathbb{F}_{1} \geq \mathbb{F}$ is a finite extension, we see that $F_{R_{1}}$ is separable over $F_{R}$ and $F_{R_{1}} \leq F_{s}$. We assume that $F_{R_{1}} \neq F_{s}$. Let $\bar{\alpha} \in F_{s}$ be such that $F_{s}=F_{R_{1}}(\bar{\alpha})$, and let $f \in R_{1}[x]$ be a unitary polynomial such that $\bar{f} \in F_{R_{1}}[x]$ is the minimal polynomial of $\bar{\alpha}$ over $F_{R_{1}}$. The element $\bar{\alpha}$ is separable over $F_{R_{1}}$. Therefore, by the Hensel lemma, there exists a root $\alpha$ of $f$ in $R_{0}$ such that $\bar{\alpha}=\alpha+\mathbf{m}\left(R_{0}\right)$. Let $F_{1}^{\prime} \rightleftharpoons F_{1}(\alpha)$ and $\mathbb{F}_{1}^{\prime}=\left\langle F_{1}^{\prime}, R_{0} \cap F_{1}^{\prime}\right\rangle$. Then $\left[F_{1}^{\prime}: F_{1}\right]=\left[F_{R_{1}}(\bar{\alpha}): F_{R_{1}}\right] \leq\left[F_{R_{1}^{\prime}}: F_{R_{1}}\right]$. Consequently, $F_{R_{1}}(\bar{\alpha})=F_{R_{1}^{\prime}}=F_{s}$, and the multiplicativity of relative degrees of extensions and ramification indices shows that $\left[\Gamma_{R_{1}^{\prime}}: \Gamma_{R}\right]=\left[\Gamma_{R_{1}}: \Gamma_{R}\right]$ and $\left[F_{1}^{\prime}: F\right]=\left[F_{1}^{\prime}: F_{1}\right]\left[F_{1}: F\right]=\left[F_{s}: F_{R_{1}}\right]\left[F_{R_{1}}: F_{R}\right]\left[\Gamma_{R_{1}^{\prime}}:\right.$ $\left.\Gamma_{R}\right]=\left[F_{R_{1}^{\prime}}: F_{R}\right]\left[F_{R_{1}^{\prime}}: \Gamma_{R}\right]$. Therefore, $\mathbb{F}_{1}^{\prime} \geq \mathbb{F}_{1}$ is a tame extension of $\mathbb{F}$ and $\mathbb{F}_{1}^{\prime} \neq \mathbb{F}_{1}$, which contradicts maximality. It follows that $F_{R_{0}}$ is a purely inseparable extension of $F_{R_{1}}\left(=F_{s}\right)$.

Now, we prove that the quotient group $\Gamma_{R_{0}} / \Gamma_{R_{1}}$ is a $p$-group. Suppose the contrary. Then there exists a prime number $q \neq p$ and an element $\gamma_{0} \in \Gamma_{R_{0}} \backslash \Gamma_{R_{1}}$ such that $q \gamma_{0} \in$ $R_{1}$. Let $a$ be an element of $F^{\times}$such that $v_{R_{0}}(a)=\gamma_{0}$. Then $v_{R_{0}}\left(a^{q}\right)=q v_{R_{0}}(a)=q \gamma_{0} \in$ $\Gamma_{R_{1}}$. We choose $b \in F_{1}^{\times}$so that $v_{R_{1}}(b)=q \gamma_{0}$. Then $v_{R_{0}}\left(a^{q} b^{-1}\right)=0$ and $0 \neq \overline{a^{q} b^{-1}} \in F_{R_{0}}$. Since $F_{R_{0}}$ is a purely inseparable extension of $F_{R_{1}}$, we see that, for an appropriate $n \in \omega$, we have $\overline{\left(a^{q} b^{-1}\right)^{p^{n}}} \in F_{R_{1}}$. Then there exists $c \in R_{1} \backslash \mathbf{m}\left(R_{1}\right)$ such that $\overline{\left(a^{q} b^{-1}\right)^{p^{n}} c^{-1}}=1$. By the Hensel lemma, the polynomial $x^{q}-\left(a^{q} b^{-1}\right)^{p^{n}} c^{-1} \in R_{0}[x]$ has a root $\alpha$ such that $\bar{\alpha}=1$. We have $\alpha^{q}=\left(a^{q} b^{-1}\right)^{p^{n}} c^{-1}$ and $b^{p^{n}} c=\left(a^{p^{n}} \alpha^{-1}\right)^{q}$; i.e., the polynomial $x^{q}-b^{p^{n}} c, b^{p^{n}} c \in F_{1}$, has a root $a^{p^{n}} \alpha^{-1}$ in $F_{0}$. We obtain $v_{R_{0}}\left(a^{p^{n}} \alpha^{-1}\right)=q^{-1} v_{R_{0}}\left(b^{p^{n}} c\right)=$ $p^{n} q^{-1} v_{R_{0}}(b)=p^{n} q^{-1} q \gamma_{0}=p^{n} \gamma_{0}$. However, since $q \neq p, \gamma_{0} \notin \Gamma_{R_{1}}$, and $q \gamma_{0} \in \Gamma_{R_{1}}$, we have $p^{n} \gamma_{0} \notin \Gamma_{R_{1}}$. Consequently, for $F_{2} \rightleftharpoons F_{1}\left(a^{p^{n}} \alpha^{-1}\right)$ we obtain $\left[F_{2}: F_{1}\right]=q$ and $\left[\Gamma_{R_{2}}: \Gamma_{R_{1}}\right]=q$, where $R_{2} \rightleftharpoons R_{0} \cap F_{2}$, which contradicts the maximality of $\mathbb{F}_{1}$.

Therefore, $\mathbb{F}_{0} \geq \mathbb{F}_{1}$ is a purely wild extension.

The proposition is proved.

We shall prove that the field $\mathbb{F}_{1}$ in Proposition 2 is unique. For this, we need more definitions.

We say that a finite extension $\mathbb{F}_{0} \geq \mathbb{F}$ is inertial if $F_{R_{0}}$ is a separable extension of $F_{R}$ and $\left[F_{0}: F\right]=\left[F_{R_{0}}: F_{R}\right]$.

An algebraic extension $\mathbb{F}_{0} \geq \mathbb{F}$ is inertial if, for every intermediate subextension $\left(\mathbb{F}_{0} \geq\right)$ $\mathbb{F}_{1} \geq \mathbb{F}$ finite over $\mathbb{F}\left(\left[F_{1}: F\right]<\omega\right)$, the extension $\mathbb{F}_{1} \geq \mathbb{F}$ is inertial.

From this definition it is clear that every inertial extension is tame. 
Corollary 6 (to the definition). Let $\mathbb{F}_{0} \geq \mathbb{F}_{1} \geq \mathbb{F}$ be a tower of extensions. The extension $\mathbb{F}_{0} \geq \mathbb{F}$ is inertial if and only if the extensions $\mathbb{F}_{0} \geq \mathbb{F}_{1}$ and $\mathbb{F}_{1} \geq \mathbb{F}$ are inertial.

Proposition 3. Let $\mathbb{F}_{0} \geq \mathbb{F}$ be an algebraic extension. Then there exists a maximal subextension $\left(\mathbb{F}_{0} \geq\right) \mathbb{F}_{0, i} \geq \mathbb{F}$ that is an inertial extension of $\mathbb{F}$; the residue field $F_{R_{0, i}}$ of $\mathbb{F}_{0, i}$ coincides with the separable closure of $F_{R}$ in $F_{R_{0}}$, and $\Gamma_{R_{0, i}}=\Gamma_{R}$.

Proof. We start with a lemma interesting in its own right.

Lemma 1. Let $\mathbb{F}^{\prime} \geq \mathbb{F}_{i} \geq \mathbb{F}, i=0,1$, be extensions such that the extension $\mathbb{F}_{0} \geq \mathbb{F}$ is inertial. Then, for $\bar{F}_{1}^{\prime} \rightleftharpoons F_{1} F_{0}$ and $\mathbb{F}_{1}^{\prime} \rightleftharpoons\left\langle F_{1}^{\prime}, R^{\prime} \cap F_{1}^{\prime}\right\rangle$, the extension $F_{1}^{\prime} \geq \mathbb{F}_{1}$ is inertial, and if $F_{R_{0}} \leq F_{R_{1}}$, then $F_{1}^{\prime}=F_{1}$ (i.e., $\mathbb{F}_{0} \leq \mathbb{F}_{1}$ ).

Essentially, this lemma was proved in the course of the proof of Proposition 2.

Corollary 7 (to Lemma 1 ). Let $\left(\mathbb{F}^{\prime} \geq\right) \mathbb{F}_{i} \geq \mathbb{F}, i=0,1$, be inertial extensions of $\mathbb{F}$. Then $\mathbb{F}_{1}^{\prime} \geq \mathbb{F}$ is an inertial extension $\left(F_{1}^{\prime} \rightleftharpoons F_{1} F_{0}\right)$.

This implies the existence of a maximal inertial extension $F_{0, i} \geq \mathbb{F}$ that is a subextension of $\mathbb{F}_{0}$. The fact that $\Gamma_{R_{0},}=\Gamma_{R}$ follows from the definition of an inertial extension. Now, we prove that $F_{R_{0, i}}$ is the separable closure of $F_{R}$ in $F_{R_{0}}$. Assuming the contrary, we can find an element $\bar{\alpha} \in F_{R_{0}} \backslash F_{R_{0, i}}$ separable over $F_{R}$. Let $f \in R[x]$ be a unitary polynomial such that $\bar{f} \in F_{R}[x]$ is the minimal polynomial of $\bar{\alpha}$ over $F_{R}$. By the Hensel lemma, the polynomial $f$ has a root $\alpha$ in $R_{0}$ such that $\bar{\alpha}=\alpha+\mathbf{m}\left(R_{0}\right)$. Putting $F_{1} \rightleftharpoons F(\alpha)$ and $\mathbb{F}_{1}=\left\langle F_{1}, R_{0} \cap F_{1}\right\rangle$, we obtain an inertial extension $\mathbb{F}_{1} \geq \mathbb{F}$. However, in this case we have $\mathbb{F}_{1} \leq \mathbb{F}_{0, i}, \alpha \in R_{0, i}$, and $\bar{\alpha} \in F_{R_{0, i}}$, a contradiction.

The proposition is proved.

We call the field $F_{0, i}\left(\mathbb{F}_{0, i}\right)$ in Proposition 3 the inertial closure of $\mathbb{F}$ in $\mathbb{F}_{0}$.

We recall the basics of the decomposition theory of normal valued field extensions (see [5, 6).

Let $\mathbb{F}_{0} \geq \mathbb{F}$ be a normal extension of valued fields, and let $G=G\left(F_{0} / F\right)$ be the group of all $F$-automorphisms of $F_{0}$. If $F_{0, s}$ is the separable closure of $F$ in $F_{0}$, then $F_{0, s}$ is a Galois extension of $F, G\left(F_{0} / F\right)=G\left(F_{0, s} / F\right)$, and $F_{0}=F_{0, s} \otimes_{F} F_{0, \text { ins }}$, where $F_{0 \text {,ins }}$ is a purely inseparable closure of $F$ in $F_{0}$.

By definition, the inertia group $G_{i}$ of a (normal) extension $\mathbb{F}_{0} \geq \mathbb{F}$ is the (closed) subgroup $\left\{\varphi \mid \varphi \in G, \forall a \in R_{0}\left(a-\varphi(a) \in \mathbf{m}\left(R_{0}\right)\right)\right\}$ of the group $G$. The group $G_{i}$ is the kernel of the natural epimorphism $\varepsilon: G=G\left(F_{0} / F\right) \rightarrow G\left(F_{R_{0}} / F_{R}\right)$ (see Proposition 1.3.3 in [1]). Therefore, $G_{i}$ is a normal subgroup of $G$.

Remark 3. The group $G\left(F_{R_{0}} / F_{R}\right)$ is naturally isomorphic to the group $G\left(F_{0, i} / F\right)$. Therefore, $F_{0, i}=F_{0, s}^{G_{i}}$ by Galois theory.

The field $F_{0, s}^{G_{i}}$ is called the inertia field of the extension $\mathbb{F}_{0} \geq \mathbb{F}$.

By definition, the ramification group $G_{r}$ of a (normal) extension $\mathbb{F}_{0} \geq \mathbb{F}$ is the subgroup $\left\{\varphi \mid \varphi \in G_{i}, \forall a \in F_{0}^{\times}\left(\varphi(a) a^{-1}-1 \in \mathbf{m}\left(R_{0}\right)\right)\right\}$ of the inertia group $G_{i}$.

The field $F_{0, s}^{G_{r}}$ is called the ramification field of the extension $\mathbb{F}_{0} \geq \mathbb{F}$.

Now, we prove two technical statements needed in the sequel.

Lemma 2. Let $\mathbb{F}_{0} \geq \mathbb{F}$ be a finite normal extension. Then the ramification group $G_{r}$ is a p-group.

Proof. Passing from $F$ to $F_{0}^{G_{r}}$, we may assume that $G_{r}=G=G\left(F_{0} / F\right)$ and $F_{0} \geq F$ is a Galois extension. Assuming that the ramification group $G_{r}$ is not a $p$-group, we can find a prime $q \neq p$ and an element $\varphi \in G_{r} \backslash\{e\}$ such that $\varphi^{q}=i d_{F_{0}}$. We consider the field $F_{1}=F_{0}^{\varphi}=\left\{a \mid a \in F_{0}, \varphi(a)=a\right\}$. Then $F_{0}$ is a Galois extension of $F_{0}^{\varphi}$ whose 
Galois group $\langle\varphi\rangle$ is cyclic of order $q$. We note that $\langle\varphi\rangle$ is also the ramification group of the extension $\mathbb{F} \geq \mathbb{F}_{1}$. Let $\alpha \in R_{0} \backslash R_{1}$. Then $F_{0}=F_{1}(\alpha)\left(F_{0} \geq F_{1}(\alpha)>F_{1}\right)$ and $q=\left[F_{0}: F_{1}\right]$. Let $f=x^{q}+a_{1} x^{q-1}+\ldots+a_{q} \in R_{1}[x]$ be the minimal polynomial of $\alpha$ over $F_{1}$. Without loss of generality, we may assume that $a_{1}=0$ (otherwise, we can replace $\alpha$ by the element $\alpha^{\prime} \rightleftharpoons \alpha+q^{-1} a_{1}$, where $\left.q^{-1} a_{1} \in R_{1}\right)$. Then the trace $\operatorname{Tr}(\alpha)$ of $\alpha$ is equal to 0 . Let $\alpha_{0} \rightleftharpoons \alpha, \alpha_{1} \rightleftharpoons \varphi(\alpha), \ldots, \alpha_{q-1} \rightleftharpoons \varphi^{q-1}(\alpha)$ be all roots of $f$. $\operatorname{Then} \operatorname{Tr}(\alpha)=\sum \alpha_{i}$. Since $\varphi^{i} \in G_{r}$, the definition of $G_{r}$ implies that $\varphi^{i}(\alpha) \alpha^{-1}-1 \in \mathbf{m}\left(R_{0}\right), i<q$, whence $v_{R_{0}}\left(\alpha_{i} \alpha^{-1}-1\right)>0$ and $\overline{\alpha_{i}, \alpha^{-1}}=1, i<q$. Therefore, $0=\alpha^{-1} \operatorname{Tr} \alpha=\sum_{i<q} \alpha_{i} \alpha^{-1}$, and passing to the residue field $F_{R_{0}}$, we obtain

$$
\overline{\alpha^{-1} \operatorname{Tr}(\alpha)}=\sum_{i<q} \overline{\alpha_{i} \alpha^{-1}}=q \neq 0 .
$$

This contradiction proves the lemma.

Lemma 3. Let $\mathbb{F}_{0} \geq \mathbb{F}$ be a finite, normal, purely wild extension. Then $\left[F_{0}: F\right]$ is a power of $p$, and the group $G=G\left(F_{0} / F\right)$ coincides with the ramification group $G_{r}$ of the normal extension $\mathbb{F}_{0} \geq \mathbb{F}$.

Proof. Passing from $\mathbb{F}_{0}$ to the separable closure $\mathbb{F}_{0, s}$ of $\mathbb{F}$ in $\mathbb{F}_{0}\left(\left[F_{1}: F_{0, s}\right]\right.$ is a power of $p$ !), we may assume that $\mathbb{F}_{0} \geq \mathbb{F}$ is a finite Galois extension. We note that the condition $\varphi(a) a^{-1}-1 \in \mathbf{m}\left(R_{0}\right)$ for $a \in F_{0}^{\times}$is equivalent to the condition $v_{R}(\varphi(a)-a)>v_{R}(a)$.

Let $\varphi \in G$. If $a \in R_{0} \backslash \mathbf{m}\left(R_{0}\right)$, then $\varphi(a)-a \in \mathbf{m}\left(R_{0}\right)$, because $F_{R_{0}}$ is a purely inseparable extension of $F_{R}$ and $\varphi$ induces the trivial $F_{R^{-}}$-automorphism of $F_{R_{0}}$. Consequently, for $a \in R_{0} \backslash \mathbf{m}\left(R_{0}\right)$ we have $\varphi(a) a^{-1}-1 \in \mathbf{m}\left(R_{0}\right)$. Let $a \in F_{0}^{\times}$. Then $v_{R_{0}}\left(a^{p^{k}}\right) \in \Gamma_{R}$ for an appropriate $k \in \omega$ (the quotient group $\Gamma_{R_{0}} / \Gamma_{R}$ is a $p$-group!). Let $b \in F$ be such that $v_{R_{0}}\left(a^{p^{k}}\right)=v_{R_{0}}(b)=v_{R}(b)$. Then $a^{p^{k}} b^{-1} \in R_{0} \backslash \mathbf{m}\left(R_{0}\right), v_{R_{0}}\left(\varphi\left(a^{p^{k}} b^{-1}\right)-\right.$ $\left.a^{p^{k}} b^{-1}\right)>0$, and $v_{R_{0}}\left(\varphi(a)^{p^{k}}-a^{p^{k}}\right)>v_{R}(b)=v_{R_{0}}\left(a^{p^{k}}\right)$. To obtain the required relation $v_{R_{0}}(\varphi(a)-a)>v_{R_{0}}(a)$, it suffices to verify that, for all $c, d \in F_{0}^{\times}$, the relation $v_{R_{0}}(c)=v_{R_{0}}(d)=v_{R_{0}}(c-d)$ implies $v_{R_{0}}\left(c^{p}\right)\left(=v_{R_{0}}\left(d^{p}\right)\right)=v\left(c^{p}-d^{p}\right)$.

If $p$ is the characteristic of $F_{0}$, this is obvious. Suppose the characteristic of $F_{0}$ is zero and $p>2$. Then

$$
(c-d)^{p}=c^{p}+\sum_{i=1}^{p-1}(-1)^{i} C_{p}^{i} c^{p-i} d^{i}-d^{p} .
$$

Observe that $p$ divides $C_{p}^{i}, 0<i<p$, and therefore, $v_{R_{0}}\left(C_{p}^{i} c^{p-i} d^{i}\right)>v_{R_{0}}\left(c^{p-i} d^{i}\right)=$ $v_{R_{0}}\left(c^{p}\right)\left(=v_{R_{0}}\left(d^{p}\right)\right) 0,1<i<p$. Hence,

$$
v_{R_{0}}\left(\sum_{i=1}^{p-1}(-1)^{i} C_{p}^{i} c^{p-i} d^{i}\right)>v_{R_{0}}\left(c^{p}\right)=v_{R_{0}}\left(d^{p}\right),
$$

and if $v_{R_{0}}\left(c^{p}-d^{p}\right)>v_{R_{0}}\left(c^{p}\right)$, then also $v_{R_{0}}\left((c-d)^{p}\right)>v_{R_{0}}\left(c^{p}\right)$. This contradicts the assumption that $v_{R_{0}}(c-d)=v_{R_{0}}(c)$.

If $p=2$, then the same argument shows that $v_{R_{0}}\left(c^{2}+d^{2}\right)=v_{R_{0}}\left(c^{2}\right)$. However, $c^{2}-d^{2}=c^{2}+d^{2}-2 d^{2}$ and $v_{R_{0}}\left(2 d^{2}\right)>v_{R_{0}}\left(d^{2}\right)=v_{R_{0}}\left(c^{2}\right)$. Thus, $v_{R_{0}}\left(c^{2}+d^{2}\right)=$ $v_{R_{0}}\left(c^{2}+d^{2}\right)=v_{R_{0}}\left(c^{2}\right)$.

This completes the proof of the lemma.

Now we prove a proposition that will imply the uniqueness of $\mathbb{F}_{1}$ from Proposition 2 in the case where $\mathbb{F}_{0} \geq \mathbb{F}$ is a (finite) normal extension.

Proposition 4. Let $\mathbb{F}_{0} \geq \mathbb{F}$ be a finite normal extension, and let $\mathbb{F}_{1}$ be the maximal tame extension of $\mathbb{F}$ in $\mathbb{F}_{0}$. Then $F_{1}$ is the ramification field of the extension $\mathbb{F}_{0} \geq \mathbb{F}$. 
By Proposition 2, the extension $\mathbb{F}_{0} \geq \mathbb{F}_{1}$ is normal and purely wild. By Lemma 3, we have $G\left(F_{0} / F_{1}\right) \leq G_{r}$, and the degree $\left[F_{0}: F_{1}\right]$ is a power of $p$. Since the extension $\mathbb{F}_{1} \geq \mathbb{F}$ is tame, the field $F_{1}$ is a separable extension of $F$ (see Corollary 4 ). Then $F_{1} \leq F_{0, s}$, and we may assume that $F_{0, s}=F_{0}$, i.e., that $F_{0}$ is a Galois extension of $F$. Let $F_{0, i}$ be the inertial closure of $F$ in $F_{0}, F_{0, i} \leq F_{1}$. We have $\left[F_{1}: F_{0, i}\right]=\left[\Gamma_{R_{1}}: \Gamma_{R_{0, i}}\right]=\left[\Gamma_{R_{1}}: \Gamma_{R}\right]$ and $p$ does not divide the index $\left[\Gamma_{R_{1}}: \Gamma_{R}\right]$. By Galois theory, $\left[F_{1}: F_{0, i}\right]=\left[G\left(F_{0} / F_{0, i}\right)\right.$ : $\left.G\left(F_{0} / F_{1}\right)\right]$, but $G\left(F_{0} / F_{0, i}\right)=G_{i}$ by the remark preceding the definition of the inertia field. Moreover, by Galois theory, $G\left(F_{0} / F_{1}\right)$ is a $p$-group. We note that, by Lemma 2 , the ramification group $G_{r}$ is a $p$-group. Since $G\left(F_{0} / F_{1}\right) \leq G_{r}, p$ does not divide the index $\left[G_{i}: G\left(F_{0} / F_{1}\right)\right]$, and $G_{r} \leq G_{i}$ is a $p$-group, it follows that $G\left(F_{0} / F_{1}\right)=G_{r}$, and $F_{1}=F_{0}^{G_{r}}\left(=F_{0, s}^{G_{r}}\right.$ in the general case) is the ramification field of the extension $\mathbb{F}_{0} \geq \mathbb{F}_{1}$.

The proposition is proved.

Corollary 8 (to the proof). $\left[F_{0}: F_{1}\right]$ is a power of $p$.

Corollary 9. Let $(\overline{\mathbb{F}} \geq) \mathbb{F}_{j} \geq \mathbb{F}, j=0,1$, be tame finite extensions. Then $\mathbb{F}^{\prime} \geq \mathbb{F}$ is a tame extension, where $F^{\prime} \rightleftharpoons F_{0} F_{1}$ and $\mathbb{F}^{\prime} \rightleftharpoons\left\langle F^{\prime}, \bar{R} \cap F^{\prime}\right\rangle$.

Proof. Indeed, let $F_{3}$ be the smallest Galois extension of $F$ containing $F^{\prime}$ ( $F^{\prime}$ is a finite separable extension of $F$ !), and let $\mathbb{F}_{3} \rightleftharpoons\left\langle F_{3}, \bar{R} \cap F_{3}\right\rangle$. Then $\mathbb{F} \leq \mathbb{F}_{0}$ and $\mathbb{F}_{1} \leq \mathbb{F}_{3}$ are separable subextensions. Consequently, if $\mathbb{F}_{0}^{\prime} \geq \mathbb{F}_{0}$ and $\mathbb{F}_{1}^{\prime} \geq \mathbb{F}_{1} \geq \mathbb{F}$ are maximal tame subextensions, then, by Proposition 4 , we have $F_{0}^{\prime}=F_{1}^{\prime}=F_{0}^{G_{r}}$, and the extension $\left(\mathbb{F}_{0}^{G_{r}} \geq\right) \mathbb{F}^{\prime} \geq \mathbb{F}$ is tame.

Corollary 10. Let $\mathbb{F}_{0} \geq \mathbb{F}$ be a finite extension, and let $\mathbb{F}_{0} \geq \mathbb{F}_{1} \geq \mathbb{F}$ be a tower of extensions such that $\mathbb{F}_{1} \geq \mathbb{F}$ is tame and $\mathbb{F}_{0} \geq \mathbb{F}_{1}$ is purely wild. Then $\mathbb{F}_{1}$ is the largest tame subextension of $\mathbb{F}$ in $\mathbb{F}_{0}$.

Corollary 11. a) If $\mathbb{F}_{0} \geq \mathbb{F}$ is a finite purely wild extension, then $\left[F_{0}: F\right]$ is a power of $p$.

b) If $\mathbb{F}_{0} \geq \mathbb{F}$ is a finite extension and $p$ does not divide $\left[F_{0}: F\right]$, then $\mathbb{F}_{0} \geq \mathbb{F}$ is a tame extension.

Proof. a) Let $\mathbb{F}_{0} \geq \mathbb{F}$ be a purely wild extension, $\mathbb{F}_{0}^{\prime} \geq \mathbb{F}_{0}$ the smallest normal extension, and $\mathbb{F}_{0, t}^{\prime}$ the largest tame subextension of $\mathbb{F}$ in $\mathbb{F}_{0}^{\prime}$. Then $\mathbb{F}_{0, t}^{\prime} \cap F_{0}$ is a tame extension of $\mathbb{F}$. Therefore, $F_{0, t}^{\prime} \cap F_{0}=F$, and $\left[F_{0}: F\right]=\left[F_{0, t}^{\prime} F_{0}: F\right]$ (see Proposition 1) divides $\left[F_{0}^{\prime}: F_{0, t}^{\prime}\right]$, but $\left[F_{0}^{\prime}: F_{0, t}^{\prime}\right]$ is a power of $p$ (see Corollary 8).

b) Let $\mathbb{F}_{0} \geq \mathbb{F}$ be a finite extension such that $p$ does not divide $\left[F_{0}: F\right]$. Let $\mathbb{F}_{1} \geq \mathbb{F}$ be the largest tame subextension of $\mathbb{F}$ in $\mathbb{F}_{0}$. Then $p$ does not divide $\left[F_{0}: F_{1}\right]$ because $\left[F_{0}: F\right]=\left[F_{0}: F_{1}\right]\left[F_{1}: F\right]$ and $\left[F_{0}: F_{1}\right]$ are powers of $p$ by part a). Hence, $F_{0}=F_{1}$.

Corollary 12 (Ostrowski lemma). If $\mathbb{F}_{0} \geq \mathbb{F}$ is a finite extension, $f \rightleftharpoons\left[F_{R_{0}}: F_{R}\right]$, and $e \rightleftharpoons\left[\Gamma_{R_{0}}: \Gamma_{R}\right]$, then $\left[F_{0}: F\right]=f \cdot e \cdot p^{k}$ for some $k \in \omega$.

Proof. Indeed, if $\mathbb{F}_{1} \geq \mathbb{F}$ is the largest tame extension of $\mathbb{F}$ in $\mathbb{F}_{0}$, then $\left[F_{1}: F\right]=f_{1} \cdot e_{1}$, where $f_{1} \rightleftharpoons\left[F_{R_{1}}: F_{R}\right], e_{1} \rightleftharpoons\left[\Gamma_{R_{1}}: \Gamma_{R}\right],\left[F_{0}: F_{1}\right]=p^{K}$ for some $K \in \omega$ (by Corollary 11 ); we have $\left[F_{R_{0}}: F_{R_{1}}\right]=p^{k_{0}},\left[\Gamma_{R_{0}}: \Gamma_{R_{1}}\right]=p^{k_{1}}$ for some $k_{0}$ and $k_{1} \in \omega$ (by the definition of a wild extension); also, $k_{0}+k_{1} \leq K, f=f_{1} \cdot p^{k_{0}}$, and $e=e_{1} \cdot p^{k_{0}}$. This implies the corollary.

From Proposition 2 and Corollary 9 we deduce the following statement.

Theorem 1. For every algebraic extension $\mathbb{F}_{0} \geq \mathbb{F}$, there exists a maximal tame subextension $\mathbb{F}_{0} \geq \mathbb{F}_{0, t} \geq \mathbb{F}$ such that $\mathbb{F}_{0} \geq \mathbb{F}_{0, t}$ is purely wild. 
Proof. Let $F_{0, t}$ be the union of all finite extensions $F_{1} \geq F$ such that $F_{0} \geq F_{1}$ and $\mathbb{F}_{1} \rightleftharpoons\left\langle F_{1}, R_{0} \cap F_{1}\right\rangle \geq \mathbb{F}$ is tame. Then $\mathbb{F}_{0, t}=\left\langle F_{0, t}, R_{0} \cap F_{0, t}\right\rangle$ is the largest tame subextension of $\mathbb{F}$ in $\mathbb{F}_{0}$. We prove that the extension $\mathbb{F}_{0} \geq \mathbb{F}_{0, t}$ is purely wild. Let $\alpha \in F_{0}$, and let $\mathbb{F}^{\prime}=\left\langle F(\alpha), R_{0} \cap F(\alpha)\right\rangle$. If $\mathbb{F}_{t}^{\prime}$ is the largest tame subextension of $\mathbb{F}$ in $\mathbb{F}^{\prime}$, then $\mathbb{F}_{t}^{\prime}=F_{0, t} \cap F^{\prime}$ and $\mathbb{F}_{t}^{\prime} \leq \mathbb{F}_{0, t}$. By Proposition 2 , the extension $\mathbb{F}^{\prime} \geq \mathbb{F}_{t}^{\prime}$ is purely wild. In particular, if $\alpha \in R_{0} \cap F^{\prime}=R^{\prime}$, then $\bar{\alpha} \rightleftharpoons \alpha+\mathbf{m}\left(R^{\prime}\right) \in F_{R^{\prime}}$ and $\bar{\alpha}^{p^{k}} \in F_{R_{t}^{\prime}} \leq F_{R_{0, t}}$ for some $k \in \omega$, and $v_{R^{\prime}}(\alpha)^{p^{k^{\prime}}} \in \Gamma_{R_{t}^{\prime}} \leq \Gamma_{R_{0, t}}$ for $\alpha \in R^{\prime} \backslash\{0\}$ and some $k^{\prime} \in \omega$. Thus, $F_{R_{0}}$ is purely inseparable over $F_{R_{0, n}}$, and the quotient group $\Gamma_{R_{0}} / \Gamma_{R_{0, t}}$ is a $p$-group; i.e., the extension $\mathbb{F}_{0} \geq \mathbb{F}_{0, t}$ is purely wild.

The theorem is proved.

The maximal tame subextension $\mathbb{F}_{0, t} \geq \mathbb{F}$ of an algebraic extension $\mathbb{F}_{0} \geq \mathbb{F}$ is called the tame closure of $\mathbb{F}$ in $\mathbb{F}_{0}$. For the tame closure we use the notation $\mathbb{F}_{0, t}\left(\left(\mathbb{F}_{0}\right)_{t}\right.$ would be more proper); for $\mathbb{F}^{\prime} \geq \mathbb{F}$ we use $\mathbb{F}_{t}^{\prime}$, etc.

Corollary 13. Let $\mathbb{F}_{0} \geq \mathbb{F}$ be an algebraic extension and $\mathbb{F}_{0, t} \geq \mathbb{F}$ the tame closure of $\mathbb{F}$ in $\mathbb{F}_{0}$. Then, for every intermediate valued field $\mathbb{F}_{0} \geq \mathbb{F}_{1} \geq \mathbb{F}$, the tame closure of $\mathbb{F}$ in $\mathbb{F}_{1}$ coincides with $\mathbb{F}_{1} \cap \mathbb{F}_{0, t}$; in particular, if $\mathbb{F}_{1} \cap \mathbb{F}_{0, t}=\mathbb{F}$, then the extension $\mathbb{F}_{1} \geq \mathbb{F}$ is purely wild.

We say that an algebraic extension $\mathbb{F}_{0} \geq \mathbb{F}$ is split if there is a subextension $\mathbb{F}_{0} \geq$ $\mathbb{F}_{w} \geq \mathbb{F}$ such that the extension $\mathbb{F}_{w} \geq \mathbb{F}$ is purely wild and $\mathbb{F}_{0} \geq \mathbb{F}_{w}$ is tame.

The following theorem of Pank (see [3]) on splitting of the algebraic closure is of great importance.

Theorem 2. Let $\overline{\mathbb{F}} \geq \mathbb{F}$, and let $\bar{F}$ be the algebraic closure of $F$. Then, for every maximal purely wild extension $\mathbb{F}_{w} \geq \mathbb{F}$ in $\overline{\mathbb{F}}$, the extension $\overline{\mathbb{F}} \geq \mathbb{F}_{w}$ is tame.

Proof. It can easily be checked that, for a valued field $\mathbb{F}_{w}$ (as in the theorem), the field $F_{R_{w}}$ is perfect (actually, $F_{R_{w}}=F_{R}^{p^{-\omega}}$ is the smallest perfect field containing $F_{R}$ ) and the group $\Gamma_{R_{w}}$ is $p$-divisible $\left(p \Gamma_{R_{w}}=\Gamma_{R_{w}}\right)$ (actually, $\Gamma_{R_{w}}$ is the smallest $p$-divisible group containing $\left.\Gamma_{R}\right)$. Indeed, if $\beta \in F_{\bar{R}} \backslash F_{R_{w}}$ and $\beta^{p} \in F_{R_{w}}$, then there is $b \in R_{w}$ such that $\beta^{p}=b+\mathbf{m}(\bar{R})$, and so $\mathbb{F}_{w}\left(b^{p-1}\right)$ is a purely wild extension of $\mathbb{F}$. We act in the same way in the case where the group $\Gamma_{R_{w}}$ is not $p$-divisible (but $\Gamma_{\bar{R}}$ is $p$-divisible!). Similarly, we check that the field $F_{w}$ is perfect.

Let $\mathbb{F}^{\prime} \geq \mathbb{F}_{w}$ be a maximal intermediate extension (which exists by Proposition 1.5.7 in 1]). By Proposition 1.5.10 in 1], the field $\mathbb{F}^{\prime}$ is algebraically complete, $F_{R^{\prime}}=F_{R_{w}}$ is perfect, and $\Gamma_{R^{\prime}}=\Gamma_{R_{w}}$ is $p$-divisible. By Corollary 2, every algebraic extension $\mathbb{F}_{0}^{\prime} \geq \mathbb{F}^{\prime}$ is tame. Let $(\overline{\mathbb{F}} \geq) \mathbb{F}_{0} \geq \mathbb{F}_{w}$ be a finite extension. If $F_{0}^{\prime} \rightleftharpoons F^{\prime} F_{w}$, then $\mathbb{F}_{0}^{\prime} \geq \mathbb{F}^{\prime}$ is a finite tame extension. Since $\mathbb{F}_{w}$ is algebraically closed in $\mathbb{F}^{\prime}$ (this follows from the fact that $\mathbb{F}_{w}$ is maximal) and $F_{w}$ is perfect, we see that $F^{\prime}$ is a regular extension of $F_{w}$ and therefore, $F_{0} \|_{F_{w}} F^{\prime}, F_{0}^{\prime}=F_{0} \otimes_{F_{w}} F^{\prime}$, and $F_{0}$ is algebraically closed in $\mathbb{F}_{0}^{\prime}$. The extension $F_{R_{0}^{\prime}} \geq F_{R^{\prime}}=F_{R_{w}}$ is finite separable, and the field $F_{R_{w}}$ is a purely inseparable extension of $F_{R}$. Therefore, there is an element $\bar{\alpha} \in F_{R_{0}^{\prime}}$ separable over $F_{R}$ and such that $F_{R_{0}^{\prime}}=F_{R_{w}}(\bar{\alpha})$. Let $f \in R[x]$ be a monic polynomial such that $\bar{f} \in F_{R}[x]$ is the minimal polynomial of $\bar{\alpha}$ over $F_{R}$ (and over $F_{R_{w}}$ ). By the Hensel lemma, there exists a root $\alpha$ of $f$ in $R_{0}$ such that $\bar{\alpha}=\alpha+\mathbf{m}\left(R_{0}^{\prime}\right)$. Then the extension $\left(\mathbb{F}_{0} \geq\right) \mathbb{F}_{w}(\alpha) \geq \mathbb{F}_{w}$ is tame (even inertial). Next, $p$ does not divide $\left[F_{0}^{\prime}: F^{\prime}\right] \cdot\left[F_{R_{0}^{\prime}}: F_{R^{\prime}}\right]^{-1}=\left[\Gamma_{R_{0}^{\prime}}: \Gamma_{R^{\prime}}\right]$, but $\left[F_{0}^{\prime}: F^{\prime}\right]=\left[F_{0}: F_{w}\right]=\left[F_{0}: F_{w}(\alpha)\right] \cdot\left[F_{w}(\alpha): F_{w}\right]$ and $\left[F_{w}(\alpha): F_{w}\right]=\left[F_{R_{0}^{\prime}}: F_{R^{\prime}}\right]$. Consequently, $\left[F_{0}: F_{w}(\alpha)\right]=\left[\Gamma_{R_{0}^{\prime}}: \Gamma_{R^{\prime}}\right]$. However, in this case the extension $\mathbb{F}_{0} \geq \mathbb{F}_{w}(\alpha)$ is tame (Corollary $11 \mathrm{~b}$ ), whence $\mathbb{F}_{0}\left(\geq F_{w}(\alpha)\right) \geq \mathbb{F}_{w}$ is tame.

The theorem is proved. 
Now, we prove a splitting criterion for a normal extension $\mathbb{F}_{0} \geq \mathbb{F}$ in terms of Galois groups.

Proposition 5. A normal extension $\mathbb{F}_{0} \geq \mathbb{F}$ is split if and only if the epimorphism

$$
\pi: G\left(F_{0} / F\right) \rightarrow G\left(F_{0, t} / F\right)
$$

is split, i.e., there exists a (closed) subgroup $G^{\prime} \leq G\left(F_{0} / F\right)$ such that $G^{\prime} \cap G_{r}=\{e\}$ and $G^{\prime} G_{r}=G\left(F_{0} / F_{1}\right)$, where $G_{r}=\operatorname{Ker} \pi$ is the ramification group of the extension $\mathbb{F}_{0} \geq \mathbb{F}$ (such a subgroup $G^{\prime}$ is called the complement to $G_{r}$ ).

Proof. We assume that the extension $\mathbb{F}_{0} \geq \mathbb{F}$ is split and $\mathbb{F}_{0} \geq \mathbb{F}^{\prime} \geq \mathbb{F}$ is a tower of extensions such that the extension $\mathbb{F}^{\prime} \geq \mathbb{F}$ is purely wild and $\mathbb{F}_{0} \geq \mathbb{F}^{\prime}$ is tame. Let $\mathbb{F}_{0, t}$ be the tame closure of $\mathbb{F}$ in $\mathbb{F}_{0}$. We prove that $F_{0}=F^{\prime} \otimes_{F} F_{0, t}$. Let $F_{0}^{\prime} \rightleftharpoons F^{\prime} F_{0, t}$. By Proposition 1, we have $F_{0}^{\prime}=F^{\prime} \otimes_{F} F_{0, t}$. Consequently, we must prove that $F_{0}^{\prime}=F_{0}$. Since $\mathbb{F}_{0} \geq \mathbb{F}_{0}^{\prime} \geq \mathbb{F}^{\prime} \geq \mathbb{F}$, we see that the extension $\mathbb{F}_{0} \geq \mathbb{F}_{0}^{\prime}$ is tame. Since $\mathbb{F}_{0} \geq \mathbb{F}_{0}^{\prime} \geq \mathbb{F}_{0, t} \geq \mathbb{F}$, the extension $\mathbb{F}_{0} \geq \mathbb{F}_{0}^{\prime}$ is purely wild. By Proposition 3 , we have $\mathbb{F}_{0}=\mathbb{F}_{0}^{\prime}$.

We put $G^{\prime} \rightleftharpoons G\left(F_{0} / F^{\prime}\right)\left(\geq G\left(F_{0} / F\right)\right)$ and prove that $G^{\prime}$ is a complement to $G_{r}$. Since $F^{\prime} \cap F_{0, t}=F\left(\mathbb{F}^{\prime} \cap \mathbb{F}_{0, t} \geq \mathbb{F}\right.$ is tame and purely wild), $F_{0, t}=\left(F_{0, s}\right)^{G_{r}}$, and $F^{\prime}=F_{0}^{G^{\prime}}\left(F^{\prime} \geq F_{0, \text { ins }}\right.$ and $\left.F^{\prime}=\left(F^{\prime} \cap F_{0, s}\right) \otimes_{F} F_{0 \text {,ins }}\right)$, we obtain $G^{\prime} G_{r}=G\left(F_{0} / F_{1}\right)$. The property $G^{\prime} \cap G_{r}=\{e\}$ follows from the fact that $F_{0}=F^{\prime} \otimes_{F} F_{0, t}$.

Conversely, suppose that the epimorphism $\pi: G\left(F_{0} / F^{\prime}\right) \rightarrow G\left(F_{0, t} / F\right)$ is split and $G^{\prime} \geq G\left(F_{0} / F_{1}\right)$ is a complement to $G_{r}$. Consider the field $F^{\prime} \rightleftharpoons F_{0}^{G^{\prime}}$ and put $\mathbb{F}^{\prime} \rightleftharpoons$ $\left\langle F^{\prime}, R_{0} \cap F^{\prime}\right\rangle$; we prove that $\mathbb{F}^{\prime} \geq \mathbb{F}$ is a wild extension. Since $F_{0, t}=F_{0, s}^{G_{r}}$ and $G_{r} G^{\prime}=$ $G\left(F_{0} / F^{\prime}\right)$, we obtain $F^{\prime} \cap F_{0, t}=F$, and, by Corollary 13 , the extension $\mathbb{F}^{\prime} \geq \mathbb{F}$ is purely wild. By Proposition 1 , the extension $\mathbb{F}_{0}^{\prime}\left(=\left\langle F_{0} F^{\prime}, R_{0} \cap F_{0} F^{\prime}\right\rangle\right) \geq \mathbb{F}^{\prime}$ is tame. It remains to note that $F_{0} F^{\prime}=F_{0}$, which follows directly from the condition $G_{r} \cap G^{\prime}=\{e\}$.

The proposition is proved.

The statement below follows from Theorem 2 and Proposition 5.

Theorem 3 (Theorem 2.2 in [3]). The absolute ramification group $G_{r}$ (= the ramification group of the extension $\overline{\mathbb{F}} \geq \mathbb{F}$ ) has a complement in $G(F)=G(\bar{F} / F)$ (the epimorphism $\pi: G(F) \rightarrow G\left(\bar{F}_{t} / F\right) \simeq G(F) / G_{r}$ is split $)$.

The next statement says that, for every finite normal extension $\mathbb{F}_{0} \geq \mathbb{F}$, there exists a "not very large" tame extension such that the extension $\mathbb{F}_{0}^{\prime} \geq \mathbb{F}$ is split $\left(F_{0}^{\prime} \rightleftharpoons F^{\prime} F_{0}\right)$.

Proposition 6. Let $\mathbb{F}_{0} \geq \mathbb{F}$ be a finite normal extension. Then there is a finite tame Galois extension $\mathbb{F}^{\prime} \geq \mathbb{F}$ such that, for $F_{0}^{\prime} \rightleftharpoons F_{0} F^{\prime}$ and $\mathbb{F}_{0}^{\prime} \rightleftharpoons\left\langle F_{0}^{\prime}, \bar{R} \cap F_{0}^{\prime}\right\rangle$, the following conditions are fulfilled:

1) $F_{0, t}^{\prime}=F^{\prime}\left(\right.$ then $\left.F_{0, t} \leq F^{\prime}\right)$;

2) if $G^{\prime} \rightleftharpoons G\left(F^{\prime} / F\right), G_{0}^{\prime} \rightleftharpoons G\left(F_{0}^{\prime} / F\right)$, and $G_{r}^{\prime}$ is the ramification group of the normal extension $\mathbb{F}_{0}^{\prime} \geq \mathbb{F}$, then $G^{\prime}$ is a complement to $G_{r}^{\prime}$ in $G_{0}^{\prime}$;

3) there exists an embedding $\eta: G^{\prime} \rightarrow G\left(F_{0} / F\right)$ such that the composition $\pi \eta: G^{\prime} \rightarrow$ $G\left(F_{0, t} / F\right)$ coincides with the restriction to $G^{\prime}$ of the composition of the epimorphism $\pi^{\prime}: G_{0}^{\prime} \rightarrow G\left(F_{0, t}^{\prime} / F\right)$ (with kernel $\left.G_{r}^{\prime}\right)$ and the restriction epimorphism $G\left(F_{0, t}^{\prime} / F\right) \rightarrow$ $G\left(F_{0, t} / F\right)$.

Proof. Let $(\overline{\mathbb{F}} \geq) \mathbb{F}_{w} \geq \mathbb{F}$ be as in Theorem 2. Let $F^{\prime} \rightleftharpoons F_{0} F_{w}$, let $\mathbb{F}_{w}^{\prime}=\left\langle F_{w}^{\prime}, \bar{R} \cap F_{w}^{\prime}\right\rangle$, and let $\mathbb{F}^{\prime}$ be the tame closure of $\mathbb{F}$ in $\mathbb{F}_{w}^{\prime}$. The proof of Theorem 2 shows that $F_{w}^{\prime}=F^{\prime} \otimes_{F} F_{w}$, and $G^{\prime} \rightleftharpoons G\left(F^{\prime} / F\right) \simeq G\left(F_{w}^{\prime} / F_{w}\right)$ is identified (via the embedding $\eta !$ ) with a subgroup of $G\left(F_{0} / F\right)$. It is easily seen that $F_{0}^{\prime}=F \otimes_{F_{0, t}}^{\prime} F_{0}$, and therefore, $\mathbb{F}_{0}^{\prime} \geq \mathbb{F}^{\prime}$ is a wild extension and $\mathbb{F}_{0, t}^{\prime}=\mathbb{F}^{\prime}$.

We omit the routine proof of statements 2) and 3). 
In conclusion, we present yet another statement on splitting.

Proposition 7. Let $\mathbb{F}_{0} \geq \mathbb{F}$ be a finite extension. Then there exists a finite purely wild extension $\mathbb{F}^{\prime} \geq \mathbb{F}$ such that, for $F_{0}^{\prime} \rightleftharpoons F^{\prime} F_{0}$ and $\mathbb{F}_{0}^{\prime}=\left\langle F_{0}^{\prime}, \bar{R} \cap F_{0}^{\prime}\right\rangle$, the extension $\mathbb{F}_{0}^{\prime} \geq \mathbb{F}^{\prime}$ is tame (and therefore, the extension $\mathbb{F}_{0}^{\prime} \geq \mathbb{F}$ is split).

Proof. Let $(\overline{\mathbb{F}} \geq) \mathbb{F}_{w} \geq \mathbb{F}$ be as in Theorem 2 , let $F_{w}^{\prime} \rightleftharpoons F_{0} F_{w}$, and let $\mathbb{F}_{w}^{\prime} \rightleftharpoons\left\langle F_{w}, \bar{R} \cap F_{w}\right\rangle$. Then $\mathbb{F}_{w}^{\prime} \geq \mathbb{F}_{w}$ is a split tame extension. If $\mathbb{F}^{\prime \prime}=\left\langle F^{\prime \prime}, \bar{R} \cap F^{\prime \prime}\right\rangle$ is the tame closure of $\mathbb{F}$ in $\mathbb{F}_{w}^{\prime}$, then $F_{w}^{\prime}=F^{\prime \prime} \otimes_{F} F_{w}$. However, $F_{w}^{\prime}=F_{0} F_{w}$. Therefore, there exists a finite extension $F^{\prime} \geq F$ in $F_{w}$ such that $F_{0} \leq F^{\prime \prime} \otimes_{F} F^{\prime}$. It is easily seen that $\mathbb{F}^{\prime}=\left\langle F^{\prime}, \bar{R} \cap F^{\prime}\right\rangle \geq \mathbb{F}$ is the required extension.

The results presented in this paper show that the classes of tame and purely wild extensions of (Henselian) valued fields play the same role as the classes of separable and purely inseparable extensions for arbitrary fields. Furthermore, for improper valued fields $\langle F, F\rangle$ these classes coincide.

For (Henselian) valued fields, there is yet another pair of classes of extensions with the same behavior, namely, the inertial extensions and the extensions $\mathbb{F}_{0} \geq \mathbb{F}$ such that $F_{R_{0}}$ is inseparable over $\mathbb{F}$. For this pair of classes of extensions, analogs of all results of the present paper are valid. In particular, we have the following version of Theorem 3.

Theorem 3'. The inertia group $G_{i}$ of the absolute Galois group $G(F)$ of the Henselian valued field $\mathbb{F}$ has a complement in $G(F)$ (or equivalently, the epimorphism $\varepsilon: G(F) \rightarrow$ $G\left(F_{R}\right)$ is split).

For the first time, this theorem appeared in the paper [7. The book 1] contains a proof of this result (see Proposition 1.5.11) known to the author since 1965. Unfortunately, an uncritical view of that proof (maximal intermediate extensions were involved in the arguments) has led to a more general formulation of Proposition 1.5.11, which (in this generality) turned out to be wrong (at the conference in Oberwolfach (Germany) in 2003, I. B. Fesenko and M. V. Bondarko called the author's attention to this fact).

\section{REFERENCES}

[1] Yu. L. Ershov, Multiply normed fields, "Nauchn. Kniga", Novosibirsk, 2000. (Russian)

[2] S. Lang, Algebra, 3rd ed., Grad. Texts in Math., vol. 211, Springer-Verlag, New York, 2002. MR.1878556 (2003e:00003)

[3] F.-V. Kuhlmann, M. Pank, and P. Roquette, Immediate and purely wild extensions of valued fields, Manuscripta Math. 55 (1986), no. 1, 39-67. MR0828410 (87d:12012)

[4] F.-V. Kuhlmann, Henselian function fields and tame fields, Heidelberg, 1994 (manuscript).

[5] O. Zariski and P. Samuel, Commutative algebra. Vol. II, Grad. Texts in Math., vol. 29, SpringerVerlag, New York - Heidelberg, 1975. MR0389876 (52:10706)

[6] A. J. Engler and A. Prestel, Valued fields, Springer-Verlag, Berlin, 2005. MR2183496 (2007a:12005)

[7] O. V. Mel'nikov and O. I. Tavgen', The absolute Galois group of a Henselian field, Dokl. Akad. Nauk BSSR 29 (1985), no. 7, 581-583, 667. (Russian) MR0801082(87a:12007)

Sobolev Institute of Mathematics, Siberian Branch of the Russian Academy of Sciences, 4 Acad. Koptyug Avenue, 630090 Novosibirsk, Russia

E-mail address: ershov@math.nsc.ru

Received 20/APR/2007

Translated by B. M. BEKKER 